\title{
Berry phase in neutrino oscillations
}

\author{
Xiao-Gang He, ${ }^{1,2, *}$ Xue-Qian Li, ${ }^{1, \dagger}$ Bruce H. J. McKellar, ${ }^{3, \ddagger}$ and Yue Zhang ${ }^{4, \S}$ \\ ${ }^{1}$ Department of Physics, Nankai University, Tianjin \\ ${ }^{2}$ NCTS/TPE, Department of Physics, National Taiwan University, Taipei \\ ${ }^{3}$ School of Physics, University of Melbourne, Parkville, Victoria, Australia \\ ${ }^{4}$ Department of Physics, Peking University, Beijing \\ (Received 18 May 2005; published 23 September 2005)
}

\begin{abstract}
We study the Berry phase in neutrino oscillations for both Dirac and Majorana neutrinos. In order to have a Berry phase, the neutrino oscillations must occur in a varying medium, the neutrino-background interactions must depend on at least two independent densities, and also there must be $C P$ violation. If the neutrino interactions with matter are mediated only by the standard model $W$ and $Z$ boson exchanges, these conditions imply that there must be at least three generations of neutrinos. The $C P$ violating Majorana phases do not play a role in generating a Berry phase. We show that a natural way to satisfy the conditions for the generation of a Berry phase is to have sterile neutrinos with active-sterile neutrino mixing, in which case at least two active and one sterile neutrinos are required. If there are additional new $C P$ violating flavor changing interactions, it is also possible to have a nonzero Berry phase with just two generations.
\end{abstract}

DOI: 10.1103/PhysRevD.72.053012

PACS numbers: 14.60.Pq, 03.65.Vf, 12.60.-i

\section{INTRODUCTION}

In the past decade, great progress has been made in the study of neutrinos. One of the most important achievements is the observation of neutrino oscillations [1]. If different species of neutrinos have different masses and also mix with each other neutrino oscillations can occur in vacuum [2] and also in matter [3]. The oscillation effects are closely related to the phases of neutrino fields.

In quantum mechanics, particle wave functions satisfy the Schrödinger equation. Once the Hamiltonian of the system is known, the evolution of a quantum system is determined. Berry has shown that if the Hamiltonian depends on time via a set of adiabatic parameters, besides the usual dynamic phase, a nondynamic phase (the Berry phase) will also be developed [4]. Neutrino oscillations in matter, where the varying matter density plays the role of the adiabatic parameters, fit in such a situation nicely. When the neutrinos move through a medium, a Berry phase is then expected to be generated.

In this work we study the conditions with which the Berry phase can be developed in neutrino oscillations when passing through a medium. We will consider Dirac and Majorana neutrinos with both active and active-sterile neutrino mixing, and also study cases with new $C P$ violating flavor changing interactions. We find that in order to generate a Berry phase,

(a) there must be $C P$ violation,

(b) but the $C P$ violating phases peculiar to Majorana neutrinos do not contribute,

\footnotetext{
*Email address: hexg@phys.ntu.edu.tw

${ }^{\dagger}$ Email address: lixq@ nankai.edu.cn

${ }^{\ddagger}$ Email address: mckellar@physics.unimelb.edu.au

${ }^{\S}$ Email address: yzhang@pku.edu.cn
}

(c) there must be three neutrino generations if neutrinos interact with the medium only through $W$ and $Z$ exchanges, and

(d) the neutrino-background matter interaction must depend on two independent densities, such as the electron and neutron densities (which requires interactions beyond the standard model, or the introduction of sterile neutrinos), or the electron and background neutrino densities (which is possible in such astronomical objects as supernovae).

We find that active and sterile neutrino mixing can provide a natural realization of all the conditions listed above due to different interactions of the $Z$ boson with active and sterile neutrinos. If there are new interactions of neutrinos with matter, it is possible to have a Berry phase with just two neutrinos.

Several authors have considered the phase properties in neutrino oscillations [5,6]. Our discussions follow Berry's definition of Berry phase in terms of a parametric dependence of the Hamiltonian, and the phase depends on motion through a loop in parameter space [4]. This is in contrast to Ref. [6] who used a different definition due to Ref. [7] which will not be discussed here.

The conditions of generating a Berry phase for Dirac neutrino oscillation in matter with the standard $W$ and $Z$ interactions have been discussed in Ref. [5]. Our results generalize the discussions in Ref. [5] to include Majorana neutrinos, active and sterile neutrino mixing, and new interactions. Specific examples which can realize all the conditions are discussed.

\section{DIRAC NEUTRINO OSCILLATION AND BERRY PHASE}

We start with the study of the Berry phase for Dirac neutrinos going step by step to identify the origin of Berry 
phase. The discussions in this section also serve to set up notations for the analysis in the other sections. Neutrino oscillations are due to the mismatch of mass and weak interaction eigenstates of the interaction Lagrangian. For Dirac neutrinos the relevant Lagrangian, in the weak eigenstate basis where the charged leptons are already in their mass eigenstate basis, is given by

$$
\begin{aligned}
L= & \bar{\nu}_{L} i \gamma^{\mu} \partial_{\mu} \nu_{L}+\bar{\nu}_{R} i \gamma^{\mu} \partial_{\mu} \nu_{R}-\bar{\nu}_{R} M \nu_{L}-\bar{\nu}_{L} M^{\dagger} \nu_{R} \\
& -\frac{g}{2 \cos \theta_{W}} \bar{f} \gamma_{\mu}\left(g_{V}^{f}-g_{A}^{f} \gamma_{5}\right) f Z^{\mu} \\
& -\frac{g}{\sqrt{2}} \bar{l}_{L} \gamma^{\mu} \nu_{L} W_{\mu}^{-}+\text {H.c. }
\end{aligned}
$$

where $g_{V}=T_{3}^{f}-2 \sin ^{2} \theta_{W} Q_{f}$ and $g_{A}=T_{3}^{f}$ with $f, T_{3}^{f}$, and $Q_{f}$ the standard model left-handed fermions and their corresponding isospin and electric charges.

The mismatch of mass and weak eigenstates means that the neutrino mass matrix $M$ is a nondiagonal matrix. $M$ can be diagonalized to a diagonal mass matrix $\hat{M}$ by a biunitary transformation, so that $M=U^{\prime} \hat{M} U^{\dagger}$. Here $U^{\prime}$ and $U$ are unitary matrices. When writing the Lagrangian in the mass eigenstate basis $\nu_{L}^{m}=U^{\dagger} \nu_{L}$ and $\nu_{R}^{m}=U^{\prime \dagger} \nu_{R}$, the form of the first four terms does not change, with $M$ replaced by $\hat{M}$, but the $W$ interaction term in the above equation changes to $-(g / \sqrt{2}) \bar{l}_{L}^{m} \gamma^{\mu} U \nu_{L}^{m}$. $U$ is the Pentecorvo, Maki, Nagakawa, Sakata (PMNS) [2] mixing matrix, and $U^{\prime}$ is the equivalent matrix for the right-handed neutrinos which plays no further role in our analysis as it does not enter the standard model interactions. By redefining the lepton field phases, for $n$ generations of leptons, the mixing matrix $U$ can be parametrized by $n(n-1) / 2$ rotation angles and $(n-1)(n-2) / 2 C P$ violating phases.

A weak eigenstate of neutrino $\nu_{i}$ of energy $E$ produced in association with a charged lepton would propagate in vacuum as

$$
\nu_{i}(t)=\sum_{j} U_{i j} e^{-i x \cdot p_{j}} \nu_{j}^{m}(0)=\sum_{j k} U_{i j} U_{k j}^{*} \nu_{k}(0) e^{-i x \cdot p_{j}}
$$

Here $p_{j}=\left(E, \vec{p}_{j}\right)$ and $|\vec{p}|=\sqrt{E^{2}-m_{j}^{2}} \approx E-m_{j}^{2} / 2 E$.

The probability amplitude for observing a $\nu_{k}$ neutrino at time $t$ after the creation of a $\nu_{i}$ neutrino, a distance $L$ away in the direction of propagation, is given by

$$
\begin{aligned}
A\left(\nu_{i} \rightarrow \nu_{k}\right) & =\sum U_{i j} U_{k j}^{*} e^{-i x \cdot p_{j}} \\
& \approx e^{-i E(t-L)} \sum U_{i j} U_{k j}^{*} e^{-i m_{j}^{2} L / 2 E} .
\end{aligned}
$$

$\left|A\left(\nu_{i} \rightarrow \nu_{k}\right)\right|^{2}$ is the transition probability for $\nu_{i}$ to $\nu_{k}$.

The phases $-x \cdot p_{j}$ in the above are usually referred to as dynamic phases. Since we follow Berry's definition of the Berry phase in terms of a parametric dependence of the Hamiltonian, and the phase depends on motion through a loop in parameter space [4], the dynamic phase $-x \cdot p$ will not contribute to a Berry phase. In order to study the Berry phase defined in Ref. [4] one must separate the effects of the dynamic phases and identify phases which depend on some slowly varying adiabatic parameters in a given system. In the case of neutrino oscillation, we find that the slowly varying matter background can be identified as the adiabatic parameter.

In medium with a finite matter density neutrino propagation is different to that in vacuum because of the interaction of neutrinos with background matter. One can obtain the equation of motion in this case by integrating out the $W$ and $Z$ in Eq. (1) obtaining the four fermion interaction term $-\sqrt{2} G_{F} \bar{\nu}_{L} \gamma^{\mu}\left(j_{\mu} N+j_{\mu}^{\prime} N^{\prime}\right) \nu_{L}$ in the Lagrangian. In the standard model $N$ and $N^{\prime}$ are diagonal matrices representing interaction of neutrinos with the medium due to $W$ and $Z$ exchange, respectively. For the purpose of discussing neutrino oscillations in the Sun and in the Earth which are unpolarized, $j_{\mu}=\bar{e} \gamma_{\mu} e$ is the electron number density current and $N=\operatorname{diag}(1,0,0)$. This is due to $W$ exchange between the neutrinos and the background electrons. $N^{\prime}$ is a unit matrix with $j_{\mu}^{\prime}=\sum_{f} \bar{f} \gamma_{\mu} f\left(T_{3}^{f}-2 Q_{f} \sin ^{2} \theta_{W}\right)$ generated by $Z$ exchange. The term proportional to $N^{\prime}$ does not affect the mixing of the active neutrinos, and will therefore be ignored until Section V. A nontrivial effect will show up there when we discuss oscillations with sterile neutrinos.

The equations of motion are given by

$$
\begin{aligned}
i \gamma^{\mu} \partial_{\mu} \nu_{L}-M^{\dagger} \nu_{R}-\sqrt{2} G_{F} j_{\mu} \gamma^{\mu} N \nu_{L} & =0, \\
i \gamma^{\mu} \partial_{\mu} \nu_{R}-M \nu_{L} & =0 .
\end{aligned}
$$

For a static case, $j_{\mu}=\rho(x) g_{\mu 0}$. Writing $\nu_{L}=$ $e^{-i E t} \psi(x)$ for fixed energy $E$ much larger than $m_{i}$, and assuming slowly varying density $(\rho E)^{-1} d \rho / d x \ll 1$, the equation of motion for $\psi$ for neutrino beam travelling along $x$ direction is given by [8]

$$
\left[E^{2}-M^{\dagger} M+\partial_{x} \partial_{x}-2 \sqrt{2} G_{F} \rho E N\right] \psi(x)=0 .
$$

We have used the approximation $\gamma^{0} \gamma^{i}\left(-i \partial_{i}\right) \psi(x) \approx$ $-E \psi(x)$ for $E \gg m_{i}$.

The above equation can be written as

$$
\begin{aligned}
& \left(i d / d x+\left(E^{2}-M^{\dagger} M-2 \sqrt{2} G_{F} \rho E N\right)^{1 / 2}\right) \\
& \quad \times\left(i d / d x-\left(E^{2}-M^{\dagger} M-2 \sqrt{2} G_{F} \rho E N\right)^{1 / 2}\right) \psi(x)=0,
\end{aligned}
$$

where the assumption of slow variation is again used. For not too large density $\rho(x)$,

$$
\begin{aligned}
& \left(E^{2}-M^{\dagger} M-2 \sqrt{2} G_{F} \rho E N\right)^{1 / 2} \\
& \quad \approx E-M^{\dagger} M / 2 E-\sqrt{2} G_{F} \rho N,
\end{aligned}
$$

and one obtains the usual equation of motion for neutrinos in matter [3], 


$$
i \frac{d}{d x} \psi(x)=-H \psi(x), \quad H=E-\frac{M^{\dagger} M}{2 E}-A N,
$$

where $A=\sqrt{2} G_{F} \rho$.

One can write $H$ in the following form

$$
\begin{aligned}
H= & \tilde{E}-\tilde{A}, \quad \tilde{E}=E-\frac{1}{2 E} \frac{m_{1}^{2}+m_{2}^{2}+m_{3}^{2}}{3}-\frac{A}{3}, \\
\tilde{A}= & \frac{1}{2 E}\left(\begin{array}{lll}
a_{11} & a_{12} & a_{13} \\
a_{12}^{*} & a_{22} & a_{23} \\
a_{13}^{*} & a_{23}^{*} & a_{33}
\end{array}\right), \\
a_{i j}= & \frac{1}{3}\left(\Delta m_{21}^{2}-\Delta m_{32}^{2}\right) \delta_{i j}-\Delta m_{21}^{2} U_{i 1} U_{j 1}^{*} \\
& +\Delta m_{32}^{2} U_{i 3} U_{j 3}^{*}+\frac{1}{3} 2 E A\left(2 \delta_{i 1} \delta_{j 1}-\delta_{i 2} \delta_{j 2}\right. \\
& \left.-\delta_{i 3} \delta_{j 3}\right) .
\end{aligned}
$$

Note that $\tilde{A}$ is a traceless Hermitian matrix.

Writing $\psi=e^{i \int_{0}^{x} \tilde{E} d x} \tilde{\psi}$, we find that $\tilde{\psi}$ satisfies the following equation of motion

$$
i \frac{d}{d x} \tilde{\psi}=\tilde{A} \tilde{\psi}
$$

For a uniform medium one obtains a solution for $\nu(t)$ similar to Eq. (2), but with $U$ replaced by the unitary matrix $\tilde{U}$ which diagonalizes $\tilde{A}$, that is, $\tilde{A}=\tilde{U} \hat{A} \tilde{U}^{\dagger}$, and $E-$ $m_{i}^{2} / 2 E$ by $\tilde{E}-\lambda_{i} / 2 E$ with $\lambda_{i} / 2 E$ being the eigenvalues of $\tilde{A}$. The eigenvalues are given by

$$
\begin{aligned}
\lambda_{1} & =2 s \cos (\theta / 3), \quad \lambda_{2}=2 s \cos (\theta / 3+2 \pi / 3), \\
\lambda_{3} & =2 s \cos (\theta / 3-2 \pi / 3), \quad \theta=\arccos \left(t^{3} / s^{3}\right), \\
2 t^{3} & =\operatorname{Det}(2 E \tilde{A}), \\
3 s^{2} & =\left|a_{12}\right|^{2}+\left|a_{13}\right|^{2}+\left|a_{23}\right|^{2}+a_{11}^{2}+a_{22}^{2}+a_{11} a_{22} .
\end{aligned}
$$

In this case all phases are dynamic again. No Berry phases are generated.

In a nonuniform medium, a Berry phase may be generated. In this case the matrix $\tilde{U}$ is now $x$ dependent through its dependence on $\rho(x)$. Writing the wave function $\tilde{\psi}(x)$ in the diagonal basis of $\tilde{A}$, we have

$$
i \frac{d}{d x} \psi^{m}=\left(\hat{A}-i \tilde{U}^{\dagger} \frac{d}{d x} \tilde{U}\right) \psi^{m},
$$

where $\psi^{m}=\tilde{U}^{\dagger} \tilde{\psi}$.

Taking the second term on the right in the above equation as perturbation, in the adiabatic approximation, one obtains

$$
\nu(t, x)_{i}=e^{-i E t} \sum_{j} \tilde{U}_{i j} e^{+i \gamma_{j}} e^{i \int_{0}^{x}\left(\tilde{E}-\lambda_{j} / 2 E\right) d x} \nu_{j}^{m}(0),
$$

where $\gamma_{j}$ is given by

$$
\gamma_{j}=i \int_{0}^{L}\left(\tilde{U}^{\dagger} \frac{d}{d x} \tilde{U}\right)_{j j} d x=i \int_{C}\left(\tilde{U}^{\dagger} \vec{\nabla} \tilde{U}\right)_{j j} d \rho .
$$

In the above we have allowed (as will be important later) for the density parameter to be multidimensional. " $\vec{\nabla}$ " is the gradient taken in density parameter space $\vec{\rho}$. The position $L$ is chosen so that $\vec{\rho}(0)=\vec{\rho}(L)$, following Berry's prescription, and $C$ is a closed curve in parameter space. This is the Berry phase in neutrino oscillation.

\section{CONDITIONS FOR A NONZERO BERRY PHASE}

Using the above definition of Berry phase in neutrino oscillation, certain conditions have to be satisfied to generate a nonzero Berry phase. We now discuss these conditions.

The matrix $\tilde{U}$ must contain complex phases in order to have a nonzero $\gamma_{j}$. Therefore there must be at least threeneutrino mixing to have a Berry phase since with twoneutrino mixing with just $W$ and $Z$ interactions, the matrix $U$ can always be made real.

A nonzero $\left(\tilde{U}^{\dagger} \nabla_{\rho} \tilde{U}\right)_{j j}$ is not a sufficient condition for a nonzero $\gamma_{j}$. It is important to realize that the matrix $\tilde{U}$ is not uniquely defined. Because the matrix $\hat{A}$ is diagonal, if $\tilde{P}=\operatorname{diag}\left(e^{i \theta_{1}}, e^{i \theta_{2}}, e^{i \theta_{3}}\right)$, then

$$
\tilde{P} \hat{A} \tilde{P}^{*}=\hat{A}, \quad \text { thus } \quad \tilde{A}=\tilde{U} \hat{A} \tilde{U}^{\dagger}=\tilde{U} \tilde{P} \hat{A} \tilde{P}^{*} \tilde{U}^{\dagger} .
$$

Thus both $\tilde{U}$ and $\tilde{U}^{\prime}=\tilde{U} \tilde{P}$ diagonalize the matrix $\tilde{A}$, but as

$$
\left(\tilde{U}^{\prime \dagger} \vec{\nabla} \tilde{U}^{\prime}\right)_{j j}=(\tilde{U} \dagger \vec{\nabla} \tilde{U})_{j j}+i \vec{\nabla} \theta_{j},
$$

after integrating over the closed loop $C$, the term proportional to $\vec{\nabla} \theta$ vanishes. Thus the Berry phase is independent of the choice of the matrix $\tilde{U}$. One may regard the transformation $\tilde{U} \rightarrow \tilde{U} P$ as a gauge transformation, and the Berry phase is a gauge independent observable.

The phase $\theta_{j}$ can be viewed as a pure gauge transformation of Berry phase. If one can find a gauge in which the Berry phase is zero even before integrating over $C$, the phase is not physical and can be gauged away.

We illustrate this phenomenon by considering the case of three generations. One way to obtain the matrix $\tilde{U}$ is to require

$$
\left(2 E \tilde{A}-\lambda_{i}\right)\left(\begin{array}{c}
\tilde{U}_{1 i} \\
\tilde{U}_{2 i} \\
\tilde{U}_{3 i}
\end{array}\right)=0 .
$$

For the $A N$ given in Eq. (7), using the first two rows in Eq. (15), we can write

$$
\left(\begin{array}{c}
\tilde{U}_{1 i} \\
\tilde{U}_{2 i} \\
\tilde{U}_{3 i}
\end{array}\right)=\frac{1}{N_{i}}\left(\begin{array}{c}
\left(a_{22}-\lambda_{i}\right) a_{13}-a_{23} a_{12} \\
\left(a_{11}-\lambda_{i}\right) a_{23}-a_{13} a_{12}^{*} \\
a_{12} a_{12}^{*}-\left(a_{22}-\lambda_{i}\right)\left(a_{11}-\lambda_{i}\right)
\end{array}\right) .
$$




$$
\begin{aligned}
\left(\tilde{U} \frac{d}{d x} \tilde{U}^{\dagger}\right)_{i i}= & -i \frac{1}{N_{i}^{2}} \operatorname{Im}\left(a_{13} a_{12}^{*} a_{23}^{*}\right) \frac{d}{d x}\left(a_{22}-a_{11}\right) \\
= & i \Delta m_{21}^{2} \Delta m_{31}^{2} \Delta m_{32}^{2} \operatorname{Im}\left(U_{21} U_{23}^{*} U_{33} U_{31}^{*}\right) \\
& \times \frac{1}{N_{i}^{2}} \frac{d}{d x}\left(a_{22}-a_{11}\right) .
\end{aligned}
$$

Since $d\left(a_{22}-a_{11}\right) / d x=-(8 \sqrt{2} / 3) E G_{F} d \rho / d x \neq 0$, it seems that a nonzero Berry phase has been generated.

On the other hand, one can also obtain $\tilde{U}_{i j}$ by using the last two rows of Eq. (15) to obtain

$$
\left(\begin{array}{c}
\tilde{U}_{1 i}^{\prime} \\
\tilde{U}_{2 i}^{\prime} \\
\tilde{U}_{3 i}^{\prime}
\end{array}\right)=\frac{1}{\tilde{N}_{i}}\left(\begin{array}{c}
a_{23} a_{23}^{*}-\left(a_{22}-\lambda_{i}\right)\left(a_{33}-\lambda_{i}\right) \\
\left(a_{33}-\lambda_{i}\right) a_{12}^{*}-a_{23} a_{13}^{*} \\
\left(a_{22}-\lambda_{i}\right) a_{13}^{*}-a_{12}^{*} a_{23}^{*}
\end{array}\right) .
$$

One then obtains

$$
\begin{aligned}
\left(\tilde{U}^{\prime} \frac{d}{d x} \tilde{U}^{\prime \dagger}\right)_{i i}= & -i \frac{1}{N_{i}^{2}} \operatorname{Im}\left(a_{13} a_{12}^{*} a_{23}^{*}\right) \frac{d}{d x}\left(a_{33}-a_{22}\right) \\
= & i \Delta m_{21}^{2} \Delta m_{31}^{2} \Delta m_{32}^{2} \operatorname{Im}\left(U_{21} U_{23}^{*} U_{33} U_{31}^{*}\right) \\
& \times \frac{1}{\tilde{N}_{i}^{2}} \frac{d}{d x}\left(a_{33}-a_{22}\right) .
\end{aligned}
$$

For the $A N$ given before, $d\left(a_{33}-a_{22}\right) / d x=0$, this gives a zero Berry phase.

However, when we integrate the nonzero result in Eq. (17) from $x=0$ to $x=L$ the result is proportional to $\rho(L)-\rho(0)$ which vanishes. No Berry phase can be generated with one varying density.

In order to generate a nonzero Berry phase, one must go to a multidimensional parameter space. This can be achieved if $a_{i i}$ depends on more than one density $\rho_{i}$. This may happen if neutrinos interact with quarks through beyond the SM interactions, or if there are sterile neutrinos mixed with active neutrinos, and therefore the oscillation in medium depends on the neutron density also. In some astronomical and cosmological circumstances, there may be significant electron neutrino densities [9], as well as background electron densities. Although the neutrinoneutrino interactions are generated by $Z$ exchange, when electron neutrinos interact with electron neutrinos both direct and exchange diagrams contribute, while when neutrinos of other flavors interact with the electron neutrino background, only the direct diagram is possible. Thus the neutrino-background neutrino interaction is not proportional to a unit matrix in $N$. In the early Universe, significant muon and tau densities may occur. For all of these cases the interaction matrix depends on more than one density. The Berry phase developed can then be written as,

$$
\gamma_{j}=i \int_{\vec{\rho}(0)}^{\vec{\rho}(L)}\left(\tilde{U}^{\dagger} \vec{\nabla} \tilde{U}\right)_{j j} \cdot d \vec{\rho} .
$$

After a cyclic motion, one obtains

$$
\begin{aligned}
\gamma_{j}= & i \oint\left(\tilde{U}^{\dagger} \vec{\nabla} \tilde{U}\right)_{j j} \cdot d \vec{\rho}=i \int_{S} \vec{\nabla} \\
& \times\left(\tilde{U}^{\dagger} \vec{\nabla} \tilde{U}\right)_{j j} \cdot d \vec{S},=i \int_{S}\left(\vec{\nabla} \tilde{U}^{\dagger} \times \vec{\nabla} \tilde{U}\right)_{j j} \cdot d \vec{S},
\end{aligned}
$$

where $\vec{S}$ indicates the area enclosed by the path $\vec{\rho}$ after a cyclic motion. Note that any pure gauge terms are eliminated since a curl is taken, and $\vec{\nabla} \times \vec{\nabla} \theta_{j}=0$. Alternatively, we may say that a nonzero value of $\gamma_{j}$ indicates that the function $\left(\tilde{U}^{\dagger} \vec{\nabla} \tilde{U}\right)_{j j}$ is not a perfect derivative. We find a nonzero Berry phase after a cyclic motion to be given by, for both of our examples in Eqs. (16) and (18),

$$
\begin{aligned}
\gamma_{i}= & \frac{2}{3} \Delta m_{21}^{2} \Delta m_{31}^{2} \Delta m_{32}^{2} \operatorname{Im}\left(U_{21} U_{23}^{*} U_{31}^{*} U_{33}\right) \\
& \times \int_{S} \frac{\lambda_{i}\left(\vec{\nabla} a_{33} \times \vec{\nabla} a_{22}\right) \cdot d \vec{S}}{\left(\lambda_{i}^{2}-s^{2}\right)^{3}} .
\end{aligned}
$$

The above result agrees with that obtained in Ref. [5].

Assuming the interaction matrix $A N$ depends on two varying independent densities, $\rho$ and $\rho^{\prime}$, with the entries $\sqrt{2} G_{F}\left(\alpha_{i i} \rho+\alpha_{i i}^{\prime} \rho^{\prime}\right)$ (traceless), we have

$\left(\vec{\nabla} a_{33} \times \vec{\nabla} a_{22}\right) \cdot d \vec{S}=\left(2 \sqrt{2} E G_{F}\right)^{2}\left(\alpha_{11} \alpha_{22}^{\prime}-\alpha_{22} \alpha_{11}^{\prime}\right) d S$.

It is clear, from the above discussions, that in order to have a nonzero value for the Berry phase $\gamma_{i}$ in neutrino oscillation, there must be at least three generations of neutrinos with nonvanishing $C P$ violating phases, and the neutrino must propagate through a background medium with which it interacts through at least two independent densities.

\section{MAJORANA NEUTRINO OSCILLATION}

We have seen in the previous discussions that $C P$ violating phase in the mixing matrix plays an important role in generating a nonzero Berry phase. In the case of Dirac neutrinos this requires that there are at least three generations of neutrinos. In the case of Majorana neutrinos, there are $C P$ violating phases even with two generations. One might naively expect a nonzero Berry phase with two generations. We now clarify whether $C P$ violating Majorana phases can play a role in generating a nonzero Berry phase. The relevant Lagrangian for Majorana neutrinos is given by

$$
\begin{aligned}
L= & \bar{\nu}_{L} i \gamma^{\mu} \partial_{\mu} \nu_{L}-\frac{1}{2} \bar{\nu}_{L}^{c} M \nu_{L}-\frac{1}{2} \bar{\nu}_{L} M^{\dagger} \nu^{c} \\
& -\frac{g}{2 \cos \theta_{W}} \bar{f} \gamma_{\mu}\left(g_{V}^{f}-g_{A}^{f} \gamma_{5}\right) f Z^{\mu} \\
& -\frac{g}{\sqrt{2}} \bar{l}_{L} \gamma^{\mu} \nu_{L} W_{\mu}^{-}+\text {H.c. },
\end{aligned}
$$

where $\nu_{L}^{c}=C \bar{\nu}_{L}^{T}$ and $C=i \gamma^{2} \gamma^{0}$. 
The mass matrix $M$ is symmetric in this case, and can be diagonalized in the following way

$$
M=\sigma^{*} U^{*} P^{*} \hat{M} P^{*} U^{\dagger} \sigma^{*},
$$

where $\sigma=\operatorname{diag}\left(e^{i \beta_{1}}, e^{i \beta_{2}}, e^{i \beta_{3}}\right)$ and $P=\operatorname{diag}\left(1, e^{i \alpha_{2}}, e^{i \alpha_{3}}\right)$ are diagonal phase matrices, and we use the notation $U^{*}$ for the complex conjugate of $U$, and $U^{\dagger}$ for the Hermitian conjugate of $U$. The phases in $\sigma$ can be absorbed by a redefinition of charged lepton fields. In the neutrino mass eigenstate basis, the charged current interaction term can be written as $-(g / \sqrt{2}) \bar{l}_{L} \gamma^{\mu} U P \nu_{L} W_{\mu}^{-}$.

When neutrinos pass through a medium, an interacting term $-\sqrt{2} G_{F} j_{\mu} \bar{\nu}_{L} \gamma^{\mu} N \nu_{L}$ needs to be added to the Lagrangian. One obtains the equations of motion as,

$$
\begin{aligned}
& i \gamma^{\mu} \partial_{\mu} \nu_{L}-M^{\dagger} \nu_{L}^{c}-\sqrt{2} G_{F} j_{\mu} \gamma^{\mu} N \nu_{L}=0, \\
& i \gamma^{\mu} \partial_{\mu} \nu_{L}^{c}-M \nu_{L}+\sqrt{2} G_{F} j_{\mu} \gamma^{\mu} N \nu_{L}^{c}=0 .
\end{aligned}
$$

Expressing the above equations in the form involving just $\nu_{L}$, we have

$$
\begin{aligned}
{\left[-\partial^{2}-M^{\dagger} M-\right.} & \sqrt{2} G_{F} i \gamma^{\mu} \partial_{\mu}\left(j_{\nu} \gamma^{\nu} N\right) \\
+ & \sqrt{2} G_{F} j_{\nu} \gamma^{\nu} M^{\dagger} N\left(M^{\dagger}\right)^{-1} \\
& \left.\times\left(i \gamma^{\mu} \partial_{\mu}-\sqrt{2} G_{F} j_{\mu} \gamma^{\mu} N\right)\right] \nu_{L}=0 .
\end{aligned}
$$

In the above we have assumed that none of the neutrinos have zero mass since we want to see if there are just two generations of neutrinos a nonzero Berry phase can be generated. With two generations, if one of the neutrinos has zero mass, the Majorana phases in $P$ can be completely removed and therefore no Berry phase can be developed. We need to discuss the case where $M^{-1}$ exists.

The first three terms in Eq. (26) are the same as the equation of motion for Dirac neutrinos. Since

$$
M^{\dagger} M=U P \hat{M}^{*} P U^{T} U^{*} P^{*} \hat{M} P^{*} U^{\dagger}=U \hat{M}^{2} U^{\dagger},
$$

the Majorana phases do not appear in the first three terms. The Majorana phases may appear in the additional two terms. However, we note that the term $\left(i \gamma^{u} \partial_{\mu}-\right.$ $\left.\sqrt{2} G_{F} \gamma^{\mu} j_{\mu} N\right) \nu_{L}$ is of order $M^{\dagger} \nu_{L}^{C}$. Compared with the third term there is a suppression factor $M / E$. For practical applications, $M / E$ is much smaller than unity and can be safely neglected. With this approximation, one therefore concludes that no effect of Majorana phases will show up in neutrino oscillations. One obtains the same equation of motion for Majorana neutrinos as for Dirac neutrinos with the same approximation: $C P$ violating Majorana phases do not play a role in neutrino oscillation [10,11]. We do not agree with the equation of motion for Majorana neutrinos obtained in Ref. [12].

\section{ACTIVE-STERILE NEUTRINO OSCILLATION}

The above discussions clearly show that in order to have a nonzero Berry phase, there must be at least three gen- erations of neutrinos no matter whether they are Dirac or Majorana neutrinos. Can the situation be changed with further modifications? In the following we consider two examples where a nonzero Berry phase can be developed with just two active neutrinos.

Our first example involves active and sterile neutrino oscillations. Light sterile neutrinos $\nu_{R}^{i}$ may be needed if the LSND result [13] for neutrino oscillation is confirmed. The Lagrangian describing light left-handed active and light sterile neutrinos is similar to Eq. (24), but with $\nu_{L}$ replaced by $\left(\nu_{L}, \nu_{R}^{c}\right)^{T}$ and the mass matrix $M$ replaced by

$$
M=\left(\begin{array}{cc}
M_{L L} & M_{D}^{T} \\
M_{D} & M_{R R}
\end{array}\right),
$$

where the different terms are defined by terms in the Lagrangian: $\quad-(1 / 2) \bar{\nu}_{L}^{c} M_{L L} \nu_{L}, \quad-\bar{\nu}_{R} M_{D} \nu_{L}, \quad$ and $-(1 / 2) \nu_{R} M_{R} \nu_{R}^{c}$.

The matrices $N$ and $N^{\prime}$ are still diagonal, with $N=$ $\operatorname{diag}(1,0,0, \ldots, 0)$, and $N^{\prime}=\operatorname{diag}\left(I_{n_{a}}, O_{n_{s}}\right)$. Here $n_{a}$ and $n_{s}$ are the numbers of the active and sterile neutrinos. The $n \times n$ matrices $I_{n}$ and $O_{n}$ are a unit matrix and a matrix with all elements equal to zero, respectively. The matrix $N$ plays the same role as discussed earlier. The matrix $N^{\prime}$ can no longer be ignored because it is not a unit matrix and will affect mixing in matter [11]. To have some specific idea on how $N^{\prime}$ affects oscillation, let us consider a simple case of neutrino oscillations with two active and one sterile neutrinos. This is effectively a three generation oscillation with the $N^{\prime}$ term included.

One can obtain the equations of motion for the present case by replacing several quantities in relevant equations. These are

(1) replacing $A N$ in Eq. (6) by $A N+B N^{\prime}$, where $B=$ $\sqrt{2} G_{F} \rho^{\prime} \quad$ with $\quad \rho^{\prime}=\sum_{f} \rho_{f}\left(T_{3}^{f}-2 \sin ^{2} \theta_{W} Q_{f}\right)=$ $\left(-1 / 2+2 \sin ^{2} \theta_{W}\right) \rho+\left(1 / 2-2 \sin ^{2} \theta_{W}\right) \rho_{p}+$ $(-1 / 2) \rho_{n}$ with $\rho, \rho_{p}$, and $\rho_{n}$ being the background electron, proton, and neutron number densities, respectively;

(2) replacing $A / 3$ in Eq. (7) by $A / 3+n_{a} B / 3$ in the expression for $\tilde{E}$

(3) replacing the term in $2 E \tilde{A}$ proportional to $A$ by $2 \sqrt{2} E G_{F}\left(A N_{i j}+B N_{i j}^{\prime}-(1 / 3)\left(A+n_{a} B\right) \delta_{i j}\right.$.

In particular,

$$
\begin{aligned}
a_{i j}= & \frac{1}{3}\left(\Delta m_{21}^{2}-\Delta m_{32}^{2}\right) \delta_{i j}-\Delta m_{21}^{2} U_{i 1} U_{j 1}^{*} \\
& +\Delta m_{32}^{2} U_{i 3} U_{j 3}^{*}+\frac{1}{3} 2 E \sqrt{2} G_{F}\left(2 \delta_{i 1} \delta_{j 1}\left(\rho+\frac{1}{2} \rho^{\prime}\right)\right. \\
& \left.-\delta_{i 2} \delta_{j 2}\left(\rho-\rho^{\prime}\right)-\delta_{i 3} \delta_{j 3}\left(1+2 \rho^{\prime}\right)\right)
\end{aligned}
$$

An interesting feature in the present case is that in the diagonal entries $a_{i i}$ of the Hamiltonian, more than one density naturally appears which is a necessary condition for a nonzero Berry phase. Assuming two active and one sterile neutrino mixing with the neutron density to be 
independent of the electron density in a neutral medium, like the Sun and the Earth, we obtain

$$
\left(\vec{\nabla} a_{33} \times \vec{\nabla} a_{22}\right) \cdot d \vec{S}=\left(2 \sqrt{2} E G_{F}\right)^{2} \frac{1}{6} d S .
$$

Here the surface is the one spanned by the densities $\left(\rho, \rho_{n}\right)$ for the closed loop " $C$."

It is possible to have a nonzero Berry phase in oscillations of two active and one sterile neutrinos, as long as there are $C P$ violating phases in the mixing matrix. One may wonder if there is a Berry phase with one active and two sterile neutrino mixing. The result is negative. In both cases, two active and one sterile, and one active and two sterile neutrino mixing cases, the mixing matrix $\tilde{U}$ contains $C P$ violating phases. However the later case has $a_{22}=a_{33}$ which results in a zero Berry phase as can be seen from Eq. (19). Obviously with more numbers of active and sterile neutrinos mixing, the conditions for generating a nonzero Berry phase can be realized.

\section{NEW INTERACTIONS}

We finally consider a case with new interactions. These interactions may be $C P$ violating and flavor changing. A possible form of interaction interesting to us is $R$-parity violating supersymmetric (SUSY) interactions [14], $\frac{1}{2} \lambda_{i j k} L_{L}^{i} L_{L}^{j} E_{R}^{C, k}, \lambda_{i j k}^{\prime} L_{L}^{i} Q_{L}^{j} D_{R}^{c k}$, where $L_{L}, E_{R}, Q_{L}$, and $D_{R}^{c}$ are the chiral lepton doublet, charged lepton singlet, quark doublet, and down quark singlet in the supersymmetric extension of SM. The quark supermultiplets are also color triplet. $i, j$, and $k$ are flavor indices. $\lambda_{i j k}$ is antisymmetric in exchanging the first two indices. This interaction will not change the PMNS mixing matrix and neutrino masses, but will change the neutrino interaction in matter. For example, exchange of right-handed sleptons and squarks can generate an interaction Lagrangian given by

$$
\begin{aligned}
L_{\text {int }}= & \frac{\lambda_{1 i k} \lambda_{1 j k}^{*}}{2 m_{\tilde{e}_{R}^{k}}^{2}} \bar{\nu}_{L}^{j} \gamma^{\mu} \nu_{L}^{i} \bar{e}_{L} \gamma_{\mu} e \\
& +\frac{\lambda_{i 1 k}^{\prime} \lambda_{j 1 k}^{\prime *}}{2 m_{\tilde{d}_{R}^{k}}^{2}} \bar{\nu}_{L}^{j} \gamma^{\mu} \nu_{L}^{i} \bar{d}_{L} \gamma_{\mu} d_{L} .
\end{aligned}
$$

The first term in the above Lagrangian has the same form as exchange of $W$ between electron and neutrino. The second term is different. When neutrinos are passing through matter, this term will generate a term proportional to the neutron and/or proton density in the Hamiltonian. The neutron density can be independent from the electron density. For the two generation case, the interaction matrix $A N$ defined earlier is modified to be

$$
A N=\left(\begin{array}{ll}
A_{11} & A_{12} \\
A_{12}^{*} & A_{22}
\end{array}\right)=\sqrt{2} G_{F}\left(\begin{array}{cc}
\rho \alpha_{11}+\rho^{\prime} \alpha_{11}^{\prime} & \rho \alpha_{12} e^{i \delta_{12}+\rho^{\prime} \alpha_{12}^{\prime} e^{i \delta_{12}^{\prime}}} \\
\rho \alpha_{12} e^{-i \delta_{12}}+\rho^{\prime} \alpha_{12}^{\prime} e^{-i \delta_{12}^{\prime}} & \rho \alpha_{22}+\rho^{\prime} \alpha_{22}^{\prime}
\end{array}\right),
$$

where $\alpha_{i j}=\left|\lambda_{1 i k} \lambda_{1 j k}^{*}\right| / 4 \sqrt{2} G_{F} m_{e^{k}}^{2}$, and $\delta_{12}$ is the phase of $\lambda_{1 i k} \lambda_{1 j k}^{*} / m_{e_{R}^{k}}^{2}$. Similarly for the primed quantities. $C P$ is violated if ${ }^{e_{R}} \sin \delta_{12} \neq 0$ or $\sin \delta_{12}^{\prime} \neq 0$. $\rho$ is the electron density in matter. $\rho^{\prime}$ includes proton and neutron densities in matter which come from the second term in the above Lagrangian. $\rho^{\prime}$ can be independent from $\rho$. There are constraints on the allowed size for the $R$-parity violating interactions [14]. $\alpha_{i j}$ and $\alpha_{i j}^{\prime}$ can be as large as a percent. Since our purpose is to demonstrate the possibility of having a nonzero Berry phase with just two neutrino generations, the actual number is not important for us.

The quantities $\tilde{E}$ and $\tilde{A}$ in Eq. (7) for the above case are given by

$$
\begin{aligned}
\tilde{E} & =E-\frac{1}{2}\left(m_{1}^{2}+m_{2}^{2}\right)-\frac{1}{2}\left(A_{11}+A_{22}\right), \\
\tilde{A} & =\frac{1}{2 E}\left(\begin{array}{cc}
a_{11} & a_{12} \\
a_{12}^{*} & -a_{11}
\end{array}\right), \\
a_{11} & =-\frac{1}{2} \Delta m_{21}^{2} \cos (2 \theta)-\frac{1}{2} 2 E\left(A_{22}-A_{11}\right), \\
a_{12} & =\frac{1}{2} \Delta m_{21}^{2} \sin (2 \theta)+2 E A_{12},
\end{aligned}
$$

where $\theta$ is the vacuum mixing angle. The eigenvalues of

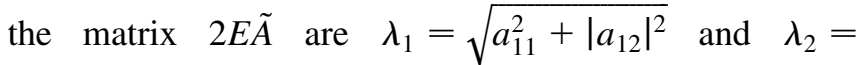
$-\sqrt{a_{11}^{2}+\left|a_{12}\right|^{2}}$.

We find

$$
\begin{aligned}
\gamma_{j}= & i \oint\left(\tilde{U}^{\dagger} \vec{\nabla} \tilde{U}\right)_{j j} \cdot d \vec{\rho}=i \oint \frac{a_{12}^{*} \vec{\nabla} a_{12}-a_{12} \vec{\nabla} a_{12}^{*}}{4 \lambda_{j}\left(\lambda_{j}-a_{11}\right)} \cdot d \vec{\rho} \\
= & -i \int_{S} \frac{1}{4 \lambda_{j}^{3}}\left[a_{11} \vec{\nabla} a_{12}^{*} \times \vec{\nabla} a_{12}+\vec{\nabla} a_{11}\right. \\
& \left.\times\left(a_{12} \vec{\nabla} a_{12}^{*}-a_{12}^{*} \vec{\nabla} a_{12}\right)\right] \cdot d \vec{S} \\
& +\frac{i}{4} \int_{S} \frac{\left|a_{12}\right|^{2}}{\lambda_{j}\left(\lambda_{j}-a_{11}\right)} \vec{\nabla} \times \frac{a_{12}^{*} \vec{\nabla} a_{12}-a_{12} \vec{\nabla} a_{12}^{*}}{\left|a_{12}\right|^{2}} \cdot d \vec{S} .
\end{aligned}
$$

To demonstrate that indeed the above case can produce a nontrivial geometric phase, let us consider a simple case where $a_{11}=a$ and $a_{12}=b e^{i \omega x}$ with constants $a$ and $b$. Integrating over one period, $x=2 \pi / \omega$, the Berry phase is given by 


$$
\begin{aligned}
& \gamma_{1}=-\pi\left(1+\frac{a}{\sqrt{a^{2}+b^{2}}}\right), \\
& \gamma_{2}=-\pi\left(1-\frac{a}{\sqrt{a^{2}+b^{2}}}\right) .
\end{aligned}
$$

Note that in the above example $C P$ is violated because $a_{12}$ is complex.

We see that a nonzero Berry phase can be developed in two neutrino oscillations if there are at least two independent varying matter densities, and also a nonzero difference in the $C P$ violating phases of the off diagonal elements in the interaction matrix $A N$. The case can be easily generalized to the case with three neutrinos.

\section{CONCLUSIONS}

We have studied Berry phase in neutrino oscillations for both Dirac and Majorana neutrinos. In order to have a nonzero Berry phase there must exist at least three generations of neutrinos with $C P$ violation in the mixing matrix and the oscillations must occur in a background with more than one varying densities if the interaction with matter is due to the standard model $W$ and $Z$ exchange. $C P$ violating Majorana phases do not play a role in generating a Berry phase.

If neutrino oscillations involve only active neutrinos, the interaction of $Z$ boson exchange does not affect neutrino oscillations in matter. If there is active and sterile neutrino mixing, the situation changes and $Z$ boson exchange does affect neutrino oscillations in matter. This scenario provides a natural setting to realize the condition of more than one density in matter since the $Z$ boson exchange is sensitive to electron and also neutron densities in matter.

With new $C P$ violating and flavor changing interactions, it is possible to have nonzero Berry phases even with just two generations, but the interactions must still depend on two independent densities in the background medium.

Experimental observation of the effects of a nonzero Berry phase in neutrino oscillation is very interesting, but it is a very difficult task, especially as it is necessary that the path of the neutrinos in position-space correspond to a closed path in the density-space. As mentioned earlier, in certain astronomical situations, the conditions on the parameters for generating a nonzero Berry phase may be realized, such as inside the Sun, neutron stars, and etc.
We denote such an object by $O$. If neutrinos from a distance source passing through $O$, and the path is such that the parameters before entering $O$ are the same as those on leaving $O$, the Berry phase effects may manifest.

On the earth, the best chance of observing the Berry phase effects in neutrino oscillations may come from long baseline neutrino oscillation experiments. The usual neutrino oscillation patterns depend on the distance $L$ via the dynamic phase difference $\Delta m^{2} L / 4 E$, but the Berry phase depends on the cyclic variation of the densities which adds an additional term $\Delta \gamma$ to the dynamic phase difference as can be seen from Eq. (11). Varying oscillation distance $L$, while maintaining the cyclicity condition one may be able to extract $\Delta \gamma$ if such a term exists.

Another possibility is to note that the dynamic phase and the Berry phase have a different dependence on the energy, so, as long as the conditions for the existence of the Berry phase are satisfied, it could be observed by determining the dependence of the oscillation amplitudes on the energy. Comparing neutrino and antineutrino oscillations in long baseline experiments can also obtain additional information. When going from neutrino to antineutrino oscillations in matter, there are two sign changes for the Berry phase generation. One sign change comes from $C P$ violating phase and another comes from the sign change in the interactions with matter densities. The effects of Berry phase will change the difference in neutrino and antineutrino oscillation rates in different ways from the usual matter and $C P$ violating effects.

Since the matter densities and changes in densities on the earth are small, the net Berry phase effects are expected to be small. It is probably very difficult to observe the effects. However, since several long baseline neutrino oscillation experiments are planned, analyses described here can be carried out and may produce interesting results.

\section{ACKNOWLEDGMENTS}

This work was supported in part by the NSC, NNSFC, and ARC. X.G. H. and B. McK. are, respectively, grateful to the School of Physics, University of Melbourne and the Departments of Physics at Nankai University and National Taiwan University for their hospitality where parts of the work reported here were done. We thank the referee for helpful suggestions.
[1] Q. R. Ahmad et al. (SNO Collaboration), Phys. Rev. Lett. 92, 181301 (2004); Phys. Rev. Lett. 89, 011301 (2002); Phys. Rev. Lett. 89, 011302 (2002); S. Fukuda et al. (Super-Kamiokande Collaboration), Phys. Lett. B 539, 179 (2002); B. T. Cleveland et al., Astrophys. J. 496, 505 (1998); R. Davis, Prog. Part. Nucl. Phys. 32, 13
(1994); D. N. Abdurashitov et al. (SAGE Collaboration), Phys. Rev. D 60, 055801 (1999); J. N. Abdurashitov et al. (SAGE Collaboration, Zh. Eksp. Teor. Fiz. 122, 211 (2002) [J. Exp. Theor. Phys. 95, 181 (2002)]; W. Hampel et al. (GALLEX Collaboration), Phys. Lett. B 447, 127 (1999); C. Cattadori (GNO Collaboration), Nucl. 
Phys. B Proc. Suppl. 111, 311 (2002); K. Eguchi et al. (Kamland Collaboration), Phys. Rev. Lett. 90, 021802 (2003); Y. Fukuda et al. (Kamiokande Collaboration), Phys. Lett. B 335, 237 (1994); R. Becker-Szendy et al. (IMB Collaboration), Nucl. Phys. (Proc. Suppl.) 38, 331 (1995); W. W. M. Allison et al. (Soudan Collaboration), Phys. Lett. B 449, 137 (1999); M. Ambrosio et al. (MACRO Collaboration), Phys. Lett. B 434, 451 (1998); M. H. Ahn et al. (K2K Collaboration), Phys. Rev. Lett. 90, 041801 (2003).

[2] B. Pentecorvo, Sov. Phys. JETP 6, 429 (1957); 7, 172 (1958); Z. Maki, M. Nakagawa, and S. Sakata, Prog. Theor. Phys. 28, 870 (1962).

[3] L. Wolfenstein, Phys. Rev. D 17, 2369 (1978); S. P. Mikheyev and A. Yu. Smirnov, Nuovo Cimento Soc. Ital. Fis. C 9, 17 (1986).

[4] M. V. Berry, Proc. R. Soc. A 392, 45 (1984).

[5] V. Naumov, Sov. Phys. JETP 74, 1 (1992); Phys. Lett. B 323, 351 (1994).
[6] M. Blasone, P. Henning, and G. Vitiello, Phys. Lett. B 466, 262 (1999); X.-B. Wang, L. C. Kwek, Y. Liu, and C. H. Oh, Phys. Rev. D 63, 053003 (2001).

[7] Y. Aharonov and J. Anandan, Phys. Rev. Lett. 58, 1593 (1987).

[8] A. Halprin, Phys. Rev. D 34, 3462 (1986).

[9] See for example D. Nortzold and G. Raffelt, Nucl. Phys. B307, 924 (1988); M. J. Thomson and B. H. J. McKellar, Phys. Lett. B 259, 113 (1991).

[10] S. Bilenky, J. Hosek, and S. Petcov, Phys. Lett. B 94, 495 (1980).

[11] P. Langacker et al., Nucl. Phys. B282, 589 (1987).

[12] J.-S. Lee, H.-M. Kwon, and J.-K. Kim, Mod. Phys. Lett. A A10, 1593 (1995).

[13] A. Aguilar et al. (LSND Collaboration), Phys. Rev. D 64, 112007 (2001); B. Armbruster et al. (Karmen Collaboration), Phys. Rev. D 65, 112001 (2002).

[14] For a recent review see: M. Chemtob, Prog. Part. Nucl. Phys. 54, 71 (2005). 